\title{
Correction to: The Purine Salvage Pathway and the Restoration of Cerebral ATP: Implications for Brain Slice Physiology and Brain Injury
}

Bruno G. Frenguelli ${ }^{1}$

Published online: 25 October 2017

(C) Springer Science+Business Media, LLC 2017

\section{Correction to: Neurochem Res}

DOI 10.1007/s11064-017-2386-6

The original version of Figure 4A contained superfluous text. This has now been removed. The correct Fig. 4 is given below:

b.g.frenguelli@warwick.ac.uk

1 School of Life Science, University of Warwick,

Coventry CV4 7AL, UK 
Fig. 4 Elevating cellular ATP with RibAde inhibits LTP via adenosine $\mathrm{A}_{1}$ Rs. a LTP induced by a tetanus $(100 \mathrm{~Hz} / 1 \mathrm{~s}$; arrow) resulted in robust LTP in standard aCSF, but steadily decremented to baseline in slices pre-incubated in RibAde. b This decrementing LTP was prevented by the adenosine $A_{1} R$ antagonist 8-CPT $(1 \mu \mathrm{M})$. Inset are fEPSPs taken before (solid lines) and at $60 \mathrm{~min}$ after the induction of LTP (dashed lines) in standard aCSF (control) and RibAde-treated slices. c-e Decreasing the number of TBS pulses $(80,40,20$, respectively) induced LTP, which is consistently lower in RibAdetreated slices. 20 TBS pulses $(0.5 \times$ TBS; $)$ failed to induce significant LTP above baseline. f Summary of LTP at $60 \mathrm{~min}$ induced in a (tetanus; open symbols), c-e. Data for 120 pulses from the LTP (at $30 \mathrm{~min}$ ) induced by $3 \times$ TBS used to evoke the adenosine release in Fig. 5A. Grey arrowheads indicate where LTP was not significantly greater than baseline in RibAde-treated slices. A twoway ANOVA (standard aCSF or RibAde treatment vs number of pulses) showed a significant effect of treatment on the LTP evoked by the various stimulation protocols $\left(\mathrm{F}_{1.51}=6.47688\right.$; $\mathrm{p}=0.014)$. Adapted from [23]
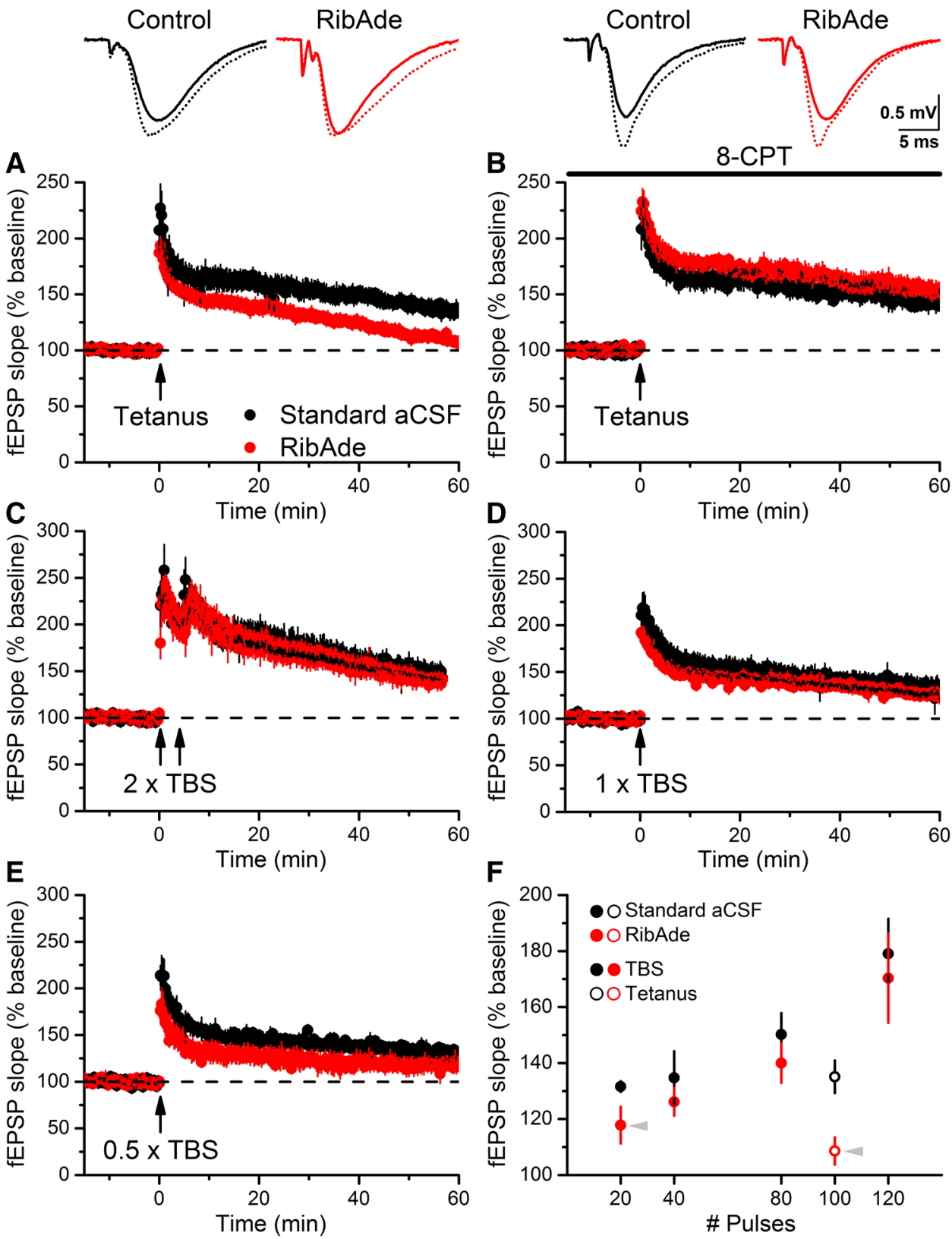